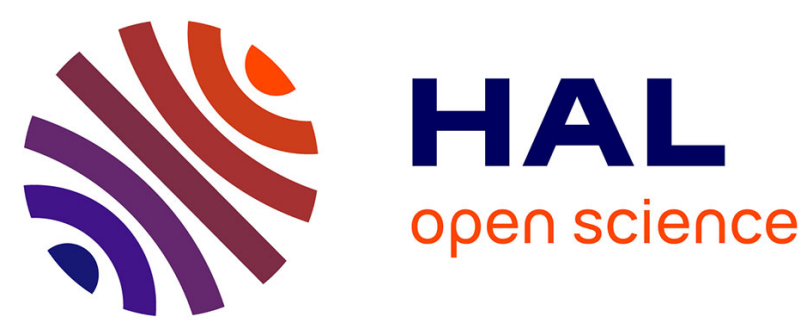

\title{
POLITIQUE PARISIENNE DES TRANSPORTS ET ACCESSIBILITE DES EMPLOIS EN ÎLE-DE-FRANCE : LE COÛT REGIONAL D'UNE POLITIQUE LOCALE
}

Nicolas Costes, Pierre Kopp, Rémy Prud'Homme

\section{To cite this version:}

Nicolas Costes, Pierre Kopp, Rémy Prud'Homme. POLITIQUE PARISIENNE DES TRANSPORTS ET ACCESSIBILITE DES EMPLOIS EN ÎLE-DE-FRANCE : LE COÛT REGIONAL D'UNE POLITIQUE LOCALE. XLVe Colloque de l'Association de Science Régionale de Langue Française (ASRDLF) - "Territoires et action publique territoriale: nouvelles ressources pour le développement régional", 2008, Rimouski, France. halshs-00318944

\section{HAL Id: halshs-00318944 \\ https://shs.hal.science/halshs-00318944}

Submitted on 5 Sep 2008

HAL is a multi-disciplinary open access archive for the deposit and dissemination of scientific research documents, whether they are published or not. The documents may come from teaching and research institutions in France or abroad, or from public or private research centers.
L'archive ouverte pluridisciplinaire HAL, est destinée au dépôt et à la diffusion de documents scientifiques de niveau recherche, publiés ou non, émanant des établissements d'enseignement et de recherche français ou étrangers, des laboratoires publics ou privés. 


\title{
POLITIQUE PARISIENNE DES TRANSPORTS ET ACCESSIBILITE DES EMPLOIS EN ÎLE-DE-FRANCE : LE COÛT REGIONAL D'UNE POLITIQUE LOCALE
}

\author{
Nicolas Costes, Pierre Kopp, Rémy Prud'homme ${ }^{1}$
}

Août 2008 - colloque ASRDLF, Rimouski, Québec, Canada.

\begin{abstract}
Résumé: Cet article évalue le coût de l'inflexion de la politique parisienne des transports intervenue dès 2001 en analysant ses effets sur l'accessibilité des emplois en Île-de-France. La diminution de l'espace viaire alloué à l'automobile dans Paris est à l'origine d'un allongement des temps de parcours domicile-travail qui modifie les conditions d'appariement de la main-d'œuvre. Une modélisation de l'accès au marché régional du travail nous permet d'estimer l'évolution du nombre moyen d'emplois accessibles en un temps de transport donné. En affectant les conditions de transport de l'ensemble des actifs franciliens transitant par Paris, nous en concluons que cette politique locale inflige un coût substantiel à la région Île-de-France.
\end{abstract}

Codes JEL :

Mots clés : accessibilité, marché du travail, transport.

"Cities can be thought of as the absence of physical space between people and firms. » Edward L. Glaeser

Depuis 2001, la municipalité parisienne s'est engagée dans une politique de refonte du Plan de Déplacement Urbain (PDU). Les objectifs affichés consistent à « redistribuer l'espace public au profit des transports en commun, des circulations dites douces (piétons, vélos, rollers)» et à « instaurer des conditions de déplacement plus justes, tout en favorisant la vitalité économique ». A cette fin, la ville opère un rationnement de l'espace viaire alloué à l'automobile de sorte à en «réduire sensiblement le trafic $»$.

Simultanément, le sous-emploi reste un problème majeur en France mais également à Paris. Le taux de chômage moyen dans Paris est supérieur à la moyenne française $(11,4 \%$ en 2004 contre $9,9 \%$ et a baissé moins vite à Paris entre 1999 et 2004 que dans le reste de la France - de $12 \%$ à $11,4 \%$ contre $12,8 \%$ à $9,9 \%$ ).

Transports et emplois sont liés. Un bon système de transport favorise les déplacements des individus vers leurs lieux de travail et élargit le périmètre de leur recherche d'emploi. Symétriquement, les entreprises, à la recherche de main-d'œuvre, bénéficient des effets du système de transport qui leur permet d'accéder à de nouveaux bassins de travailleurs. Le système de transport favorise donc l'appariement entre l'offre et la demande de travail (Cervero, 2001). En raccourcissant les temps de transports, l'espace des appariements possibles augmente, le marché du travail est donc plus efficace et l'emploi facilité. L'emploi n'est qu'un des éléments positivement affecté par les transports. Ce sont en fait toutes les interactions, y compris celles qui ne passent pas par le marché (échange de connaissances, rencontres, etc.) qui bénéficient de la mobilité.

\footnotetext{
${ }^{1}$ Respectivement Doctorant à l'Université Paris I (Panthéon-Sorbonne) CES Matisse et membre du Programme Doctoral ESSEC, Professeur à l'Université de Paris I (Panthéon-Sorbonne) et Professeur émérite à l'Université Paris XII.
} 
Le présent article se contente d'examiner dans quelle mesure la stratégie municipale, dans le domaine des transports, est ou non favorable à la fluidification du marché du travail et à l'augmentation de la productivité dans l'Île-de-France et à Paris. Il s'appuie sur le concept de « taille effective du marché de l'emploi » introduit par Prud'homme et Lee (1999) sur le cas de villes françaises et coréennes, et appliqué aux cas de Sao Paulo (Prud'homme 1999) et de Buenos Aires (Prud'homme, Huntzinger et Kopp, 2004).

Nous proposons d'analyser les conditions d'accès à l'emploi dans la région Île-de-France et d'examiner leur évolution entre 2000 et 2004. Faute de données ultérieures, notre analyse doit être interrompue en 2004.

Nous dresserons dans une première section un rapide diagnostic des déplacements en Île-de-France. Après avoir présenté la méthodologie employée, nous estimerons la taille effective du marché régional du travail en 2004 et son évolution par rapport à 2000 (section II). Les résultats obtenus nous permettront enfin d'évaluer le coût induit par la politique parisienne des transports à l'échelle régionale (section III).

\section{I - ANALYSE DES DEPLACEMENTS EN ÎLE-DE-FRANCE}

Chaque jour, plus de 6,5 millions de déplacements pour motif de travail sont effectués par les franciliens, dont près de 2,4 millions en transports en commun $(36,1 \%)$ et 3,3 millions en voiture $(49,5 \%)^{2}$.

Le comportement des franciliens vis-à-vis des modes de transport varie selon leur secteur de résidence (tableau 1). Le flux de déplacements effectués en transports en commun décroît avec la distance à Paris et s'explique par la forte densité du réseau de transport public au cœur de l'agglomération.

Tableau 1. Nombre de déplacements pour motif de travail par zone de résidence et mode de transport (2001)

\begin{tabular}{lcccccc}
\hline & $\begin{array}{c}\text { Nombre de déplacements } \\
\text { (milliers) } \\
\text { Total }\end{array}$ & $\%$ & $T C$ & $\%$ & $V P$ & $\%$ \\
\hline Paris & 1200 & 18,3 & 681 & 71 & 278 & 29 \\
Petite Couronne & 2400 & 36,6 & 937 & 45,6 & 1118 & 54,4 \\
Grande & 2960 & 45,1 & 752 & 28,8 & 1856 & 71,2 \\
Couronne & 6560 & 100 & 2370 & 36,1 & 3252 & 49,5 \\
Île-de-France & & & & & & \\
\hline
\end{tabular}

Source : DREIF, Enquête Globale de Transport 2002

Le tableau 1 indique que $45,1 \%$ des flux de déplacements déclenchés par un motif lié au travail sont imputables aux actifs résidant en grande couronne ${ }^{3}(2,96$ millions de déplacements) et $36,6 \%$ à ceux de la petite couronne $4(2,4$ millions de déplacements). Les actifs parisiens ne contribuent qu'à hauteur de 18,3\% (1,2 million) du total des déplacements pendulaires (domicile-travail) en Île-de-France.

Près de 2,4 millions $(36,3 \%)$ de déplacements pour motif de travail sont de type radial et ont Paris comme ville de départ ou de destination (tableau 2). Parmi eux, 0,78 million

\footnotetext{
${ }^{2}$ Les $14,4 \%$ restants se répartissent entre la marche $(0,67$ million de déplacements, $10,2 \%)$, les deux roues $(0,26$ million de déplacements soit $4 \%$ du total) et les autres modes mécanisés $(0,01$ million, $0,2 \%)$.

${ }^{3} \mathrm{La}$ «Grande Couronne » est composée des départements de la Seine-et-Marne, des Yvelines, de l'Essonne et du Val-d'Oise.

${ }^{4}$ La «Petite Couronne» regroupe les départements des Hauts-de-Seine, de la Seine-Saint-Denis et du Val-de-Marne.
} 
$(32,8 \%)$ sont effectués intra-muros, 1,04 million (43,7\%) relient Paris et une commune de la petite couronne et 0,56 million $(23,5 \%)$ Paris et une commune de la grande couronne.

Les liaisons périphériques transversales se répartissent entre les déplacements internes à la petite couronne (1,46 millions soit $22,3 \%$ du total) et à la grande couronne $(1,83$ million, $27,9 \%$ du total). Les liaisons entre la grande et la petite couronne représentent $13,1 \%$ du nombre total de déplacements effectués pour motif de travail ( 0,86 million).

Tableau 2. Répartition du nombre de déplacements pour motif de travail entre les zones de résidence

\begin{tabular}{ccc}
\hline & Nombre de liaisons (milliers) & $\%$ \\
\hline Paris $<>$ Paris & 780 & 32,8 \\
Paris $<>$ Petite Couronne & 1040 & 43,7 \\
Paris $<>$ Grande Couronne & 560 & 23,5 \\
\cline { 2 - 3 } & 2380 & 100 \\
PC $<>$ PC & 1460 & - \\
PC $<>$ GC & 860 & - \\
GC $<>$ GC & 1830 & - \\
Hors Île-de-France & 30 & - \\
\hline
\end{tabular}

Source : DREIF, Enquête Globale de Transport 2002

La portée ${ }^{5}$ moyenne des déplacements pour motif de travail en Île-de-France s'élève à $12,4 \mathrm{Km}$ en transports en commun contre 9,7 $\mathrm{Km}$ en voiture. Elle varie de façon substantielle selon le lieu de résidence, le secteur de destination et le mode de transport utilisé (tableau 3).

Tableau 3. Portée des déplacements pour motif de travail

\begin{tabular}{ccccc}
\hline Liaison & \multicolumn{2}{c}{ Portée $(\mathrm{Km})$} & \multicolumn{2}{c}{ Distance Effective $(\mathrm{Km})$} \\
& TC & VP & TC & VP \\
Paris & 3,9 & 3,7 & 5 & 4,8 \\
Paris > Paris & 9,4 & 8,1 & 12,2 & 10,5 \\
Paris > PC & 25,0 & 22,7 & 32,5 & 29,5 \\
Paris $>$ GC & 12,8 & 11,5 & 16,6 & 14,9 \\
Moyenne & & & & 10,7 \\
Petite Couronne & 9,5 & 8,2 & 12,3 & 7,4 \\
PC > Paris & 8,3 & 5,7 & 10,8 & 21,6 \\
PC > PC & 21,1 & 16,6 & 27,4 & 13,3 \\
PC $>$ GC & 13,0 & 10,2 & 16,9 & 28,6 \\
Moyenne & & & & 22 \\
Grande Couronne & 24,9 & 22,0 & 32,4 & 11,3 \\
GC > Paris & 21,5 & 16,9 & 27,9 & 20,7 \\
GC > PC & 10,1 & 8,7 & 13,1 & 12,6 \\
GC $>$ GC & 18,8 & 15,9 & 24,4 & \\
Moyenne & 12,4 & 9,7 & 16,1 & \\
Ille-de-France & & & & \\
\hline
\end{tabular}

Source : DREIF, Enquête Globale de Transport 2002

L'ensemble de ces données, descriptives des conditions d'accès à l'emploi en Île-de-France, souligne les limites d'une approche locale, intra départementale, des déplacements pour motif de travail. Elles posent par ailleurs la question de l'impact

\footnotetext{
${ }^{5}$ La portée est exprimée en kilomètre parcouru à vol d'oiseau. Cette unité de mesure est utilisée dans la plupart des bases de données relatives au transport et aux déplacements. Elle se distingue de la distance effective parcourue sur les différents réseaux terrestres (routes, chemin de fer). Nous multiplions la portée par le coefficient retenu dans la littérature $(1,3)$ pour calculer les distances effectives.
} 
régional d'actions localisées susceptibles de modifier les conditions d'appariement de la main-d'œuvre.

Dans cette optique, nous devons nous interroger sur les effets de l'inflexion de la politique parisienne des transports sur l'accessibilité des emplois à l'échelle de la région Île-de-France.

\section{II - MARCHE DE L'EMPLOI THEORIQUE ET EFFFECTIF EN ÎLE-DE-FRANCE}

La région Île-de-France dispose d'un marché du travail de plus de cinq millions d'emplois, dont 1,65 million dans Paris. Il est toutefois nécessaire de distinguer la taille du marché théorique et le nombre d'emplois auxquels les actifs ont réellement accès, autrement dit, sa taille effective.

En matière de déplacement, les décisions individuelles sont guidées par la distance du trajet ou le temps de transport induit. Compte tenu du niveau de développement des infrastructures de transport en Île-de-France, une analyse fondée sur la géographie physique, exprimée en kilomètres parcourus, serait peu pertinente. La représentation anamorphique $^{6}$ de la région selon les durées de déplacement de commune à commune révèle en effet une géographie du temps très différente de la géographie physique. Selon l'offre de transport disponible et les caractéristiques géographiques de l'espace parcouru, le temps nécessaire pour parcourir un kilomètre peut varier de manière substantielle à l'échelle de la région. Nous avons donc retenu une approche où les temps de parcours constituent la variable de décision du déplacement.

En nous situant du point de vue des actifs, la taille effective du marché du travail telle que nous la calculons décrit donc le nombre moyen d'emplois potentiels qu'un actif peut prétendre occuper selon sa commune de résidence pour un temps de transport donné, en considérant ces emplois comme disponibles.

La taille effective du marché du travail se mesure également du point de vue des entreprises. Elle correspond au nombre d'actifs dont une entreprise peut potentiellement utiliser les services, en fonction du temps de transport pour l'atteindre.

La mesure que nous proposons est établie à partir de la somme des emplois déjà occupés par d'autres actifs et suppose une parfaite substituabilité entre les différents types d'emplois offerts.

1 - La taille effective du marché du travail en 2004

L'objet de cette section est de mesurer le nombre d'emplois auxquels un actif peut prétendre postuler, selon sa commune de résidence. Pour ce faire, il convient d'identifier les moyens de transport disponibles pour se rendre d'une commune à l'autre.

Le calcul de la taille effective du marché du travail (TEMT) exige de croiser trois bases de données. Les deux premières, issues du Recensement Général de la Population de l'INSEE ${ }^{8}$, décrivent respectivement, le nombre d'emplois « au lieu de travail» et le nombre d'actifs occupés par communes d'Île-de-France.

$\mathrm{Au}$ sens de l'INSEE, l'emploi «au lieu de travail» comprend «l'ensemble des personnes qui ont une profession et qui l'exercent sur le territoire français au moment

\footnotetext{
${ }^{6}$ Le principe de l'anamorphose repose sur la déformation d'un fond de carte selon une donnée statistique précise, le temps de parcours dans le cas qui nous intéresse.

${ }^{8}$ INSEE (1999) « Recensement Général de la Population ».
} 
du recensement ». Cet emploi est comptabilisé dans la commune du lieu de travail. La "population active occupée » comprend les personnes qui "déclarent exercer une profession (salariée ou non) même à temps partiel, aider un membre de la famille dans son travail (même sans rémunération), être apprenti ou stagiaire rémunéré ».

Ces deux bases ont été croisées avec les matrices de temps de transport par mode, élaborées en 2004 par la Direction Régionale de l'Equipement d'Île-de-France (DREIF/PoD/DESD). Ces matrices proposent une modélisation des temps de parcours aux heures de pointe pour les différents modes de transport utilisés. Nous retiendrons ici les liaisons effectuées en transports en commun (TC) et en voiture (VP).

\section{1 - L’approche méthodologique}

L'exploitation des matrices de temps de transport par mode, élaborées par la DREIF, nous a permis d'établir les périmètres d'emplois accessibles par commune de résidence.

Du fait de notre base de données, notre champ d'analyse est strictement restreint aux limites administratives de la région Île-de-France ce qui implique que nous ne tenions pas compte du fait que les actifs franciliens peuvent avoir accès aux emplois d'autres départements. De même, nous négligeons le fait que certains emplois d'île-de-France puissent être occupés par des actifs d'autres régions. Enfin, les données relatives au temps de transport reposent sur un découpage de la région Île-de-France en zones communales, reprenant les limites administratives de chaque commune, en zones supra communales, regroupant plusieurs communes attenantes, ou en zones infra communales résultantes d'une subdivision de certaines communes, généralement les plus étendues et les plus peuplées. L'exploitation de ces données et leur croisement avec celles de l'INSEE a donc exigé un travail préalable d'homogénéisation. La région Île-de-France a ainsi été découpée en 566 zones distinctes ${ }^{10}$.

La modélisation des temps de parcours effectuée par la DREIF repose sur les portées entre chaque zone, autrement dit sur la distance à vol d'oiseau qui les sépare. Afín de proposer une mesure précise de la taille effective du marché du travail, les temps de parcours ont été recalculés en appliquant le coefficient auquel nous avons fait référence plus haut ${ }^{11}$.

Les données les plus récentes relatives à l'emploi et au nombre d'actifs à l'échelle locale sont fournies par le Recensement Général de la Population de $1999^{12}$.

L'absence de bases actualisées à l'échelle locale ne remet toutefois pas en cause la pertinence de notre mesure compte tenu des évolutions observées depuis.

Après une évolution positive, jusqu'en 2001, la population active occupée d'Île-de-France a diminué pour atteindre en 2003 un niveau très proche de celui de 1999 (4,7 millions contre 4,8 millions en 1999).

Cette stabilité ne nous dispense cependant pas de faire l'hypothèse selon laquelle la distribution spatiale des actifs occupés et des emplois est semblable à celle de 1999 (tableau 4). La localisation des emplois et des actifs observée dans le passé est susceptible de se transformer à l'avenir. Notre hypothèse de stabilité revient cependant à minimiser les effets de cette évolution sur l'accessibilité des emplois.

\footnotetext{
${ }^{10}$ Les données sources de la DREIF reposent sur un découpage de la région Île-de-France en 1277 zones, sur la base desquelles a été établie la matrice des temps de parcours.

${ }^{11}$ Voir note 5.

12 INSEE (1999) « Recensement Général de la Population ».
} 
Cette simplification conduit à sous-estimer une évolution positive de l'accessibilité des emplois.

Tableau 4. Répartition de la population active occupée d'Île-de-France en 1999

\begin{tabular}{lcc}
\hline & Nb d'emplois & $\%$ \\
\hline Paris & $\mathbf{9 9 1 ~ 0 0 3}$ & $\mathbf{2 0 , 4}$ \\
& & \\
Petite Couronne & $\mathbf{1 7 5 0 4 0 7}$ & $\mathbf{3 6 , 1}$ \\
Hauts-de-Seine & 651004 & 13,4 \\
Seine-Saint-Denis & 556628 & 11,5 \\
Val-de-Marne & 542775 & 11,2 \\
& & \\
Grande Couronne & $\mathbf{2 1 0 5} \mathbf{1 5 4}$ & $\mathbf{4 3 , 4}$ \\
Seine-et-Marne & 525786 & 10,8 \\
Yvelines & 598757 & 12,4 \\
Essonne & $507 \mathbf{7 2 2}$ & 10,5 \\
Val-d'Oise & 472889 & 9,8 \\
& & \\
île-de-France & $\mathbf{4 8 4 6 5 6 4}$ & $\mathbf{1 0 0}$ \\
\hline Source $:$ INSEE & &
\end{tabular}

D'après le dernier recensement exhaustif, l'emploi en Île-de-France a évolué positivement pour atteindre 5,34 millions ${ }^{13}$ en 2004 contre 5,04 millions en 1999. Cette augmentation de $6 \%$ n'a toutefois pas bénéficié de façon homogène aux huit départements. Ce sont ainsi les départements de la Seine-Saint-Denis et du Val-de-Marne en petite couronne et de la Seine-et-Marne et du Val-d'Oise en grande couronne qui ont le plus bénéficié de cette évolution (tableau 5).

Tableau 5. Evolution de l'emploi régional entre 1999 et 2004

\begin{tabular}{lccccc}
\hline & \multicolumn{2}{c}{ Nombre d'emplois } & \multicolumn{2}{c}{ Evolution } & \multicolumn{2}{c}{ Répartition (\%) } \\
& 1999 & 2004 & & $1999 / 2004(\%)$ & 2004 \\
\hline Paris & 1601 & 1650 & 3,1 & 31,8 & 30,9 \\
Hauts-de-Seine & 815 & 848 & 4,0 & 16,2 & 15,9 \\
Seine-Saint-Denis & 484 & 530 & 9,5 & 9,6 & 9,9 \\
Val-de-Marne & 472 & 510 & 8,1 & 9,4 & 9,5 \\
Petite couronne & 1771 & 1888 & 6,6 & 35,1 & 35,3 \\
Seine-et-Marne & 389 & 431 & & & 7,7 \\
Yvelines & 504 & 534 & 6,0 & 10,0 & 8,1 \\
Essonne & 401 & 422 & 5,2 & 8,0 & 7,9 \\
Val-d'Oise & 374 & 420 & 12,3 & 7,4 & 7,9 \\
Grande couronne & 1668 & 1807 & 8,3 & 33,1 & 33,8 \\
Île-de-France & 5040 & 5345 & 6,1 & 100 & 100 \\
\hline Note: & & & & &
\end{tabular}

Note : nombre d'emplois exprimé en milliers.

Source : données INSEE

L'absence de données détaillées à l'échelle communale ne nous permet pas d'effectuer une répartition actualisée de l'emploi pour chacune des 566 zones que nous avons retenues. Notre évaluation de la taille effective du marché du travail sera donc quelque peu minorée, principalement pour les zones appartenant à ces quatre départements.

${ }^{13}$ Source : INSEE, estimations annuelles d'emploi, chiffres provisoires au 31 décembre 2004 
Nous supposerons donc que les valeurs retenues reflètent la réalité du marché francilien de l'emploi sur la période étudiée (2000-2004).

Nous prenons ici le parti de nous concentrer sur la mesure de l'accessibilité des emplois et non de la main-d'œuvre.

\section{2 - La formalisation}

Nous adoptons la méthodologie développée par Prud'homme et Lee (1999) afin de déterminer les niveaux d'accessibilité des emplois en nous situant du point de vue des actifs.

Nous considérons une agglomération divisée en $n$ zones telles que $n \in\{1,2, \ldots i, \ldots j, \ldots n\}$ avec $i$ la zone d'origine ou zone de résidence et $j$ la zone de destination pour motif de travail. Dans le cas de l'Île-de-France, nous disposons de $n=566$ zones, soit $i=j=566$.

Un actif aura ainsi la possibilité d'aller travailler dans l'une des 565 zones connexes à sa zone de résidence mais il pourra également travailler et résider au sein de la même zone.

Nous notons $T_{i j}$, le temps de transport effectif nécessaire à l'appariement de la main-d'œuvre entre la zone $i$ et la zone $j, W_{i}$ le nombre d'actifs occupés résidant dans chacune des $n$ zones et $\mathrm{W}=\sum_{\mathrm{i}=1}^{\mathrm{n}} \mathrm{W}_{\mathrm{i}}$ le nombre total d'actifs occupés au sein de l'agglomération. $P_{j}$ correspond au nombre d'emplois au lieu de travail pour chaque zone de destination avec $\mathrm{P}=\sum_{\mathrm{j}=1}^{\mathrm{n}} \mathrm{P}_{\mathrm{j}}$ le nombre total d'emplois offerts.

La taille effective du marché du travail d'une zone $i$ en un temps donné $t$ de transport sera donnée par $\mathrm{L}_{\mathrm{i}}(\mathrm{t})=\sum_{\mathrm{j}} \mathrm{P}_{\mathrm{j}}$ pour tout $j$ tel que $\mathrm{T}_{\mathrm{ij}} \leq \mathrm{t}$, le temps de transport entre $i$ et $j$ est inférieur ou égal à la contrainte temporelle $t . \mathrm{L}_{\mathrm{i}}(\mathrm{t})$ est égale à la somme des emplois offerts dans les zones $j$ auxquels un actif résidant dans la zone $i$ peut prétendre à en $t$ minutes de transport.

Au niveau régional, la taille effective du marché du travail est donnée par :

$$
\mathrm{L}(\mathrm{t})=\frac{\sum_{\mathrm{i}=1}^{\mathrm{n}} \mathrm{L}_{\mathrm{i}}(\mathrm{t}) * \mathrm{~W}_{\mathrm{i}}}{\mathrm{W}}=\frac{\sum_{\mathrm{i}=1}^{\mathrm{n}} \sum_{\mathrm{j}} \mathrm{P}_{\mathrm{j}} * \mathrm{~W}_{\mathrm{i}}}{\mathrm{W}}
$$

pour tout $j$ tel que $\mathrm{T}_{\mathrm{ij}} \leq \mathrm{t}$.

Elle correspond à la moyenne des tailles effectives de chaque zone, pondérée par la part respective d'actifs qui y résident.

1.3 - L'estimation de la taille effective du marché du travail d'Île-deFrance en voiture en 2004

Comme l'indique le tableau 6 , en quarante minutes de transport, un candidat à l'emploi circulant en voiture avait, en moyenne, accès à plus de 1,3 million d'emplois, correspondant à plus d'un quart $(25,9 \%)$ de l'emploi total offert en Île-de-France. 
Tableau 6. Taille effective du marché du travail d'Île-de-France pour un parcours effectué en voiture, 2004.

\begin{tabular}{ccc}
\hline $\begin{array}{c}\text { Temps de transport } \\
\text { (minutes) }\end{array}$ & Nombre d'emplois accessibles & \% de l'emploi régional total \\
\hline 10 & 80490 & 1,6 \\
20 & 315117 & 6,2 \\
30 & 725503 & 14,4 \\
40 & 1304508 & 25,9 \\
50 & 1967002 & 39,0 \\
60 & 2650428 & 52,6 \\
90 & 4125244 & 81,8 \\
\hline
\end{tabular}

Source : calculs des auteurs

Compte tenu des contraintes spatio-temporelles, les résultats ci-dessus indiquent que la taille effective du marché du travail ne correspond qu'à une fraction du nombre total d'emplois offerts. L'accès à la totalité des emplois théoriques offerts exigeait, en 2004, que le candidat consacre au transport un temps moyen supérieur à quatre heures.

2 - La situation en 2000 et l'évolution de la taille effective du marché du travail

Le champ d'application de la politique parisienne des transports est strictement délimité par les frontières administratives de la ville. Elle a toutefois un effet majeur sur l'accessibilité globale des emplois, à l'échelle régionale. En plus des déplacements effectués par les seuls Parisiens, nous devons tenir compte des déplacements effectués par l'ensemble des actifs franciliens et ayant Paris pour origine et/ou destination.

\section{1 - L'impact de la vitesse de circulation dans Paris}

Pour apprécier l'impact réel de la politique engagée depuis 2001, il faudrait comparer la situation actuelle non pas à celle d'avant 2001, mais à la situation que nous aurions dû observer, en l'absence d'inflexion de la politique. Il est évidemment très délicat de raisonner de la sorte car il est impossible de reconstituer un tel scénario contrefactuel. Nous avons donc pris le parti de comparer la situation de 2004 avec celle de l'année 2000.

Néanmoins, afin de neutraliser les effets potentiels d'éléments exogènes sur l'évolution des temps de transport de zone à zone (construction d'infrastructures nouvelles de transport, par exemple), il nous faut raisonner, a priori, sur la base de temps de transport de zone à zone constants. Nous postulons donc que les temps de transport de l'année 2000 sont identiques à ceux dont nous disposons pour 2004.

Nous reprenons dans un premier temps les conclusions de Prud'homme, Kopp et Bocarejo (2006) selon lesquelles la vitesse moyenne de circulation automobile dans Paris a diminué de $12 \%$ entre 2000 et $2004^{14}$.

En réalité, la vitesse qui nous intéresse ici n'est pas uniquement celle à laquelle roulent les véhicules. C'est celle qui est obtenue en divisant la distance de l'origine à la destination d'un déplacement par le temps mis pour ce déplacement, y compris les temps d'accès à l'origine et à la destination. Les difficultés accrues de stationnement, qui font partie de la politique suivie, par la diminution du nombre de places de

\footnotetext{
${ }^{14}$ Cette estimation est fondée sur le rôle de l'élasticité de la demande de transport automobile urbain au prix au kilomètre, qui intègre le coût monétaire et le coût en temps. Les auteurs calculent les diminutions de vitesse associées à une diminution de la circulation. Ils montrent que, sous l'hypothèse d'absence d'amélioration d'une offre alternative de transport, la diminution de la circulation s'explique par la diminution de la vitesse et l'augmentation du coût en temps qu'elle induit.
} 
stationnement en surface et l'accroissement du «temps de recherche ${ }^{15}$ » associé, ont donc pour effet de réduire la vitesse moyenne.

Toutefois, nous faisons l'hypothèse que cette baisse ne concerne que la partie du déplacement considéré effectuée au sein des limites administratives de la ville de Paris. Nous ne tenons pas compte d'un éventuel effet de débordement de la baisse de la vitesse de circulation dans Paris aux communes limitrophes. La vitesse et donc les temps de parcours de la banlieue jusqu'aux portes de Paris et des portes de Paris vers la banlieue sont supposés constants. Cette hypothèse nous conduit à considérer nos estimations à venir comme basses.

Les données relatives au temps de parcours fournies par la DREIF ne nous permettent cependant pas de distinguer la durée effective du parcours entre les portes de Paris et les zones intra muros.

Nous avons donc pris le parti d'affecter à chaque déplacement incluant un passage dans Paris une distance forfaitaire parcourue dans la capitale de cinq kilomètres. Cette hypothèse s'appuie sur la portée (distance en ligne droite) des déplacements intra muros pour motif de travail effectués par les actifs parisiens qui s'élève à 3,7 kilomètres, soit près de cinq kilomètres effectifs. Nous avons ensuite testé la sensibilité de nos résultats à un changement de ce paramètre en considérant une distance de trois puis de sept kilomètres. Nous proposerons également un second scenarii en considérant une diminution inférieure de la vitesse de circulation.

Il ressort de nos calculs qu'en 2004, il fallait plus de dix-neuf minutes pour effectuer cinq kilomètres dans Paris contre un peu plus de dix-sept minutes en $2000^{16}$. Ces deux minutes supplémentaires, qui correspondent à une diminution de $12 \%$ de la vitesse de circulation parisienne, devront donc être retranchées des temps de parcours de 2004 pour les zones strictement parisiennes afin d'obtenir ceux de 2000, toutes choses égales par ailleurs.

Nous appliquerons le même raisonnement pour nos évaluations effectuées sur la base de parcours de trois puis de sept kilomètres.

2.2 - L'évolution de la taille effective du marché du travail pour les trajets effectués en voiture

Les données du tableau 7 montrent que la baisse de la vitesse moyenne de circulation dans Paris affecte l'accessibilité des emplois au niveau régional.

Nous retrouvons en cela les préceptes de la conjecture de Zahavi (Zahavi, 1979 ; Zahavi et Talvitie, 1980) selon laquelle l'espace pratiqué dépend non pas de la distance mais de la vitesse des déplacements. A temps de transport constant, l'augmentation de la vitesse permet d'accroître la portée spatiale des déplacements. Un actif aura ainsi accès à un nombre accru de communes et donc à davantage d'emplois en consacrant le même budget temps à ses déplacements.

Entre 2000 et 2004, pour un parcours d'une durée de trente minutes ${ }^{17}$ effectué en voiture, plus de 64600 emplois ont été rendus inaccessibles du fait de la diminution de la vitesse moyenne de circulation et de l'allongement induit des temps de transport. La

\footnotetext{
${ }^{15}$ Le « temps de recherche » s'entend comme la durée moyenne consacrée à la recherche d'une place de stationnement autorisée disponible.

16 19'36' 'secondes en 2004 contre 17'24', en 2000.

${ }^{17}$ Nous retenons un temps de trente minutes qui correspond au temps moyen des déplacements pour motif de travail effectués en voiture en Île-de-France.
} 
réduction de l'espace viaire alloué à l'automobile dans Paris est donc à l'origine d'une diminution de la taille effective du marché du travail de $8,2 \%$ par rapport à 2000 , pratiquement sans substitut de mode de transport possible puisque, comme nous le verrons, les transports en commun sont presque toujours moins performants en termes d'accessibilité aux emplois offerte.

Tableau 7. Évolution de la taille effective du marché francilien du travail en voiture (Hypothèse 1:5 Km effectués dans Paris)

\begin{tabular}{ccccc}
$\begin{array}{c}\text { Temps de } \\
\text { transport } \\
\text { (minutes) }\end{array}$ & $\begin{array}{c}\text { Nombre d'emplois } \\
\text { accessibles* }\end{array}$ & $\begin{array}{c}\text { \% de l'emploi } \\
\text { total régional }\end{array}$ & $\begin{array}{c}\text { Nombre } \\
\text { d'emplois } \\
\text { accessibles }\end{array}$ & $\begin{array}{c}\text { \% de l'emploi } \\
\text { total régional }\end{array}$ \\
\hline 10 & 93626 & 1,9 & 80490 & 1,6 \\
20 & 361281 & 7,2 & 315117 & 6,2 \\
30 & 790106 & 15,7 & 725503 & 14,4 \\
40 & 1395829 & 27,7 & 1304508 & 25,9 \\
50 & 2053326 & 40,7 & 1967002 & 39,0 \\
60 & 2714456 & 53,8 & 2650428 & 52,6 \\
90 & 4148749 & 82,3 & 4125244 & 81,8 \\
\hline
\end{tabular}

Note : * L'emploi par zone de destination est évalué à partir des données du RGP de 1999, dernière source disponible à ce niveau de détail. Nous faisons l'hypothèse que l'emploi est resté constant sur la période étudiée.

Source : calculs des auteurs

La perte d'emplois est encore plus importante lorsque nous faisons l'hypothèse que le déplacement pour motif de travail inclut un parcours de sept kilomètres dans Paris (tableau 8).

Tableau 8. Évolution de la taille effective du marché francilien du travail en voiture (Hypothèse $2: 7 \mathrm{Km}$ effectués dans Paris)

\begin{tabular}{ccccc}
\hline $\begin{array}{c}\text { Temps de } \\
\text { transport } \\
\text { (minutes) }\end{array}$ & $\begin{array}{c}\text { Nombre d'emplois } \\
\text { accessibles* }\end{array}$ & $\begin{array}{c}\text { \% de l'emploi total } \\
\text { régional }\end{array}$ & $\begin{array}{c}\text { Nombre d'emplois } \\
\text { accessibles }\end{array}$ & $\begin{array}{c}\text { \% de l'emploi } \\
\text { total régional }\end{array}$ \\
\hline 10 & 111574 & 2,2 & 80490 & 1,6 \\
20 & 377383 & 7,5 & 315117 & 6,2 \\
30 & 820414 & 16,3 & 725503 & 14,4 \\
40 & 1428027 & 28,3 & 1304508 & 25,9 \\
50 & 2087644 & 41,4 & 1967002 & 39,0 \\
60 & 2742379 & 54,4 & 2650428 & 52,6 \\
90 & 4155917 & 82,4 & 4125244 & 81,8 \\
\hline
\end{tabular}

Source : calculs des auteurs

Les candidats à l'emploi effectuant un trajet d'une durée égale à trente minutes ont accès à près de 95000 emplois de moins qu'en 2000, soit une diminution de $11,6 \%$ de la taille effective du marché du travail.

Sous l'hypothèse basse que seuls trois kilomètres effectifs seraient parcourus dans Paris, la perte d'emplois accessibles en 2004 est égale à plus de 42000 emplois, soit une diminution de 5,5\% de la taille effective du marché du travail par rapport à 2000 (tableau 9). 
Tableau 9. Évolution de la taille effective du marché francilien du travail en voiture (Hypothèse $3: 3 \mathrm{Km}$ effectués dans Paris)

\begin{tabular}{ccccc}
$\begin{array}{c}\text { Temps de } \\
\text { transport } \\
\text { (minutes) }\end{array}$ & $\begin{array}{c}\text { Nombre d'emplois } \\
\text { accessibles* }\end{array}$ & $\begin{array}{c}\text { \% de l'emploi total } \\
\text { régional }\end{array}$ & $\begin{array}{c}\text { Nombre d'emplois } \\
\text { accessibles }\end{array}$ & $\begin{array}{c}\text { \% de l'emploi } \\
\text { total régional }\end{array}$ \\
\hline 10 & 89304 & 1,8 & 80490 & 1,6 \\
20 & 342301 & 6,8 & 315117 & 6,2 \\
30 & 767977 & 15,2 & 725503 & 14,4 \\
40 & 1358871 & 26,9 & 1304508 & 25,9 \\
50 & 2014225 & 39,9 & 1967002 & 39,0 \\
60 & 2696535 & 53,39 & 2650428 & 52,6 \\
90 & 4138720 & 82 & 4125244 & 81,8 \\
\hline
\end{tabular}

Source : calculs des auteurs

3 - Le marché de l'emploi et les transports en commun

L'évolution du potentiel d'accès à l'emploi régional en fonction des temps de parcours (graphique 1) traduit le décalage de performance des modes de transport estimée à travers leur capacité à assurer une accessibilité aux emplois en un temps donné.

Graphique 1. Taille effective du marché du travail de la région île-de-France selon le mode et le temps de transport, 2004.

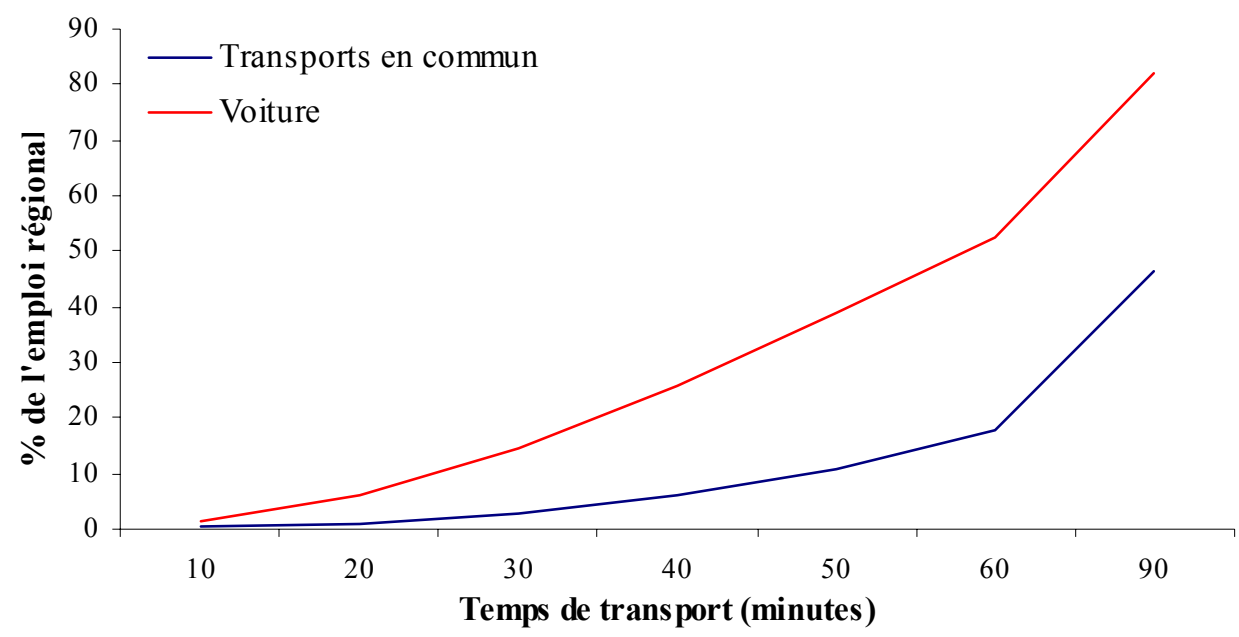

L'usage de la voiture offre en moyenne aux actifs franciliens l'accès potentiel au nombre d'emploi le plus élevé, quelle que soit la durée de transport envisagée. Pour un parcours d'une heure, l'usage de la voiture permet d'avoir potentiellement accès à près de trois fois plus d'emplois que les transports en commun.

En considérant un parcours de trente-six minutes correspondant à la durée moyenne des déplacements pour motif de travail des franciliens tous modes confondus ${ }^{18}$, 1 'usage de la voiture permet aux actifs d'avoir en moyenne accès à $20,7 \%$ des emplois $(1,04$ millions d'emplois) contre seulement 4,4\% (224 518 emplois) pour les transports en commun.

${ }^{18}$ Source : les déplacements des franciliens en 2001-2002, Enquête Globale de Transport. 
Tableau 10. Taille effective du marché du travail d'île-de-France en transports en commun

\begin{tabular}{ccc}
\hline $\begin{array}{c}\text { Temps de transport } \\
\text { (minutes) }\end{array}$ & $\begin{array}{c}\text { Nombre d'emplois } \\
\text { accessibles }\end{array}$ & \% de l'emploi total régional \\
\hline 10 & 30455 & 0,6 \\
20 & 48979 & 1,0 \\
30 & 131588 & 2,6 \\
40 & 301044 & 6,0 \\
50 & 547199 & 10,8 \\
60 & 887086 & 17,6 \\
90 & 2328524 & 46,2 \\
\hline
\end{tabular}

Source : calculs des auteurs

Une analyse désagrégée, à l'échelle départementale, souligne l'hétérogénéité des situations au sein des couronnes périphériques.

Les résultats du tableau 11 confirment la nette supériorité de la voiture en termes d'emplois rendus potentiellement accessibles, même au sein de Paris qui bénéficie pourtant d'un réseau de transport en commun très dense.

Le différentiel de performance des modes de transport s'exprime à travers le nombre d'emplois accessibles. Un parcours d'une heure effectué en voiture offre aux actifs résidant en petite couronne un accès à plus de trois millions d'emplois, avec des valeurs proches de celles observées à partir de Paris.

Pour un même parcours effectué en transports en commun, le nombre d'emplois accessibles n'est plus que de 1,39 million à partir des Hauts-de-Seine (soit 55\% de moins qu'à partir de Paris) et est inférieur à 800000 à partir de la Seine-Saint-Denis et du Val-de-Marne.

Ces résultats soulignent par ailleurs les inégalités d'accès à l'emploi selon le lieu de résidence des actifs.

En petite couronne, le fait de résider dans le département des Hauts-de-Seine (92) permet d'avoir accès jusqu'à 71,5\% de l'emploi régional en une heure de transport en voiture, contre respectivement $65,9 \%$ et $63,9 \%$ pour la Seine-Saint-Denis (93) et le Val-de-Marne (94).

En grande couronne, le Val-d'Oise (95) offre l'accessibilité potentielle aux emplois la plus importante (42,4\% de l'emploi francilien), alors qu'à peine $17,2 \%$ de l'emploi régional est accessible à partir de la Seine-et-Marne (77).

Quels que soient la durée et le mode de transport, la Seine-et-Marne est le département au sein duquel l'accès à l'emploi régional est le plus contraint. 
Tableau 11. Taille effective du marché du travail d'Île-de-France par département, mode et temps de transport

\begin{tabular}{|c|c|c|c|c|c|c|c|c|}
\hline \multirow[b]{3}{*}{$\begin{array}{c}\text { Temps de } \\
\text { transport (minutes) }\end{array}$} & \multicolumn{4}{|c|}{ Nombre moyen d'emplois accessibles } & \multicolumn{4}{|c|}{$\%$ de l'emploi total IDF } \\
\hline & \multicolumn{2}{|c|}{ Transports en Commun } & \multicolumn{2}{|c|}{ Voiture } & \multicolumn{2}{|c|}{$\begin{array}{c}\text { Transports en } \\
\text { Commun }\end{array}$} & \multicolumn{2}{|c|}{ Voiture } \\
\hline & 30 & 60 & 30 & 60 & 30 & 60 & 30 & 60 \\
\hline Paris & 465949 & 2163269 & 1464777 & 3720251 & 9,2 & 42,9 & 29,0 & $\mathbf{7 3 , 8}$ \\
\hline Petite Couronne & 72400 & 996600 & 852585 & 3395258 & 1,4 & 19,8 & 16,9 & 67,3 \\
\hline 92 & 121503 & 1391807 & 1161764 & 3604427 & 2,4 & 27,6 & 23,0 & 71,5 \\
\hline 93 & 32169 & 730146 & 589658 & 3321052 & 0,6 & 14,5 & 11,7 & 65,9 \\
\hline 94 & 46078 & 795842 & 694006 & 3220480 & 0,9 & 15,8 & 13,8 & 63,9 \\
\hline $\begin{array}{l}\text { Grande } \\
\text { Couronne }\end{array}$ & 21609 & 195263 & 227605 & 1527493 & 0,4 & 3,9 & 4,5 & 30,3 \\
\hline 77 & 11873 & 57367 & 103612 & 868045 & 0,2 & 1,1 & 2,1 & 17,2 \\
\hline 78 & 29552 & 267771 & 231278 & 1414593 & 0,6 & 5,3 & 4,6 & 28,1 \\
\hline 91 & 23342 & 167635 & 254231 & 1774328 & 0,5 & 3,3 & 5,0 & 35,2 \\
\hline 95 & 20514 & 286438 & 332227 & 2138639 & 0,4 & 5,7 & 6,6 & 42,4 \\
\hline Île-de-France & 131588 & 887086 & 725503 & 2650428 & 2,6 & 17,6 & 14,4 & 52,6 \\
\hline
\end{tabular}

La sous performance enregistrée par les transports en commun, par rapport à la voiture, en termes d'accessibilité aux emplois offerte peut s'expliquer par les différentes composantes du temps prises en compte pour évaluer le temps global de déplacement et par la structure des réseaux.

Au-delà des temps de transport et de correspondance, l'usage des transports en commun nécessite que soit intégré, dans la mesure du temps global de déplacement, les temps de rabattement sur les gares du réseau structurant, de même que les temps d'attente au départ et d'accès au lieu de destination. Associés aux ruptures de charges entre les modes de transport utilisés, ces éléments conduisent à altérer la compétitivité des transports collectifs par rapport à la voiture particulière. Il s'agit bien entendu là de moyennes. Pour la plupart des parcours, le temps de déplacement est bien plus court en voiture qu'en transports en commun, même si le contraire est vrai pour certains trajets, à certaines heures, notamment dans Paris intra-muros.

L'écart entre les niveaux d'accessibilité offerts par les modes de transport souligne également les insuffisances des infrastructures de transports publics. De manière générale, les résultats obtenus soulignent une inadaptation de l'offre de transports en commun (fréquence, liaisons proposées, etc.) à la demande. Par la densité du réseau routier existant en Île-de-France, la voiture demeure le mode de transport offrant le choix de destinations possibles le plus large en un temps donné. La structure radiale du réseau structurant de transports en commun contraint quant à elle les déplacements strictement périphériques et limite de fait la taille effective du marché du travail.

\section{III - LE COÛT DE L'INACCESSIBILITE DES EMPLOIS}

L'effet taille de marché est souvent considéré comme jouant en faveur de l'adéquation entre les besoins des offreurs et demandeurs de travail. Plus le marché du travail est vaste, plus la probabilité que les profils respectifs des actifs et des emplois coïncident serait grande (Simpson, 1992 ; Acemoglu, 1996 ; Cervero, 1996). Cette 
adéquation reste néanmoins conditionnée à l'accessibilité respective des emplois et des actifs. Comme nous l'avons vu, la taille effective du marché du travail est inférieure à sa taille théorique.

L'efficacité régionale du système de transport dépend du temps de parcours entre les lieux d'emplois et de résidence résultant de l'offre de transport et de la localisation des actifs et des entreprises.

Par sa contribution à la limitation des coûts d'appariement de la main-d'œuvre, le système de transport tend à accroître la productivité du travail. Par ailleurs, à budget temps de transport constant, l'amélioration des conditions de transport permet d'élargir l'éventail de choix offerts aux actifs en termes d'emplois et de cibler le mieux adapté à leur qualification respective.

Cette approche nouvelle de l'efficacité urbaine, fondée sur la taille effective du marché du travail plus que sur sa taille intrinsèque, est relayée par les études empirique de Prud'homme \& Lee (1999) qui concluent à l'existence d'une relation significative entre l'accessibilité des emplois et la productivité du travail approximée par le PIB par emploi.

La première, menée sur trois villes coréennes, révèle une élasticité de 0,24 pour un temps de transport de soixante minutes. Cela suggère qu'une variation de $1 \%$ de la taille effective du marché du travail s'accompagne d'une variation de $0,24 \%$ de la productivité du travail.

Une étude plus robuste portant sur vingt deux villes françaises (hors Paris) a permis de valider et d'affiner ces résultats. Les régressions laissent apparaître des élasticités allant de 0,15 à 0,24 selon les temps de parcours envisagés, de sorte qu'une élasticité moyenne de 0,2 a été retenue par les auteurs.

En nous appuyant sur ces travaux et d'après nos résultats précédents relatifs à l'évolution moyenne de l'accessibilité des emplois, nous pouvons estimer le coût de la politique parisienne des transports (tableau 12).

Tableau 12. Coût lié à l'évolution de l'accessibilité des emplois

\begin{tabular}{ccccccc}
\hline \multicolumn{5}{c}{ Hypothèse Vitesse $-12 \%$} & \multicolumn{3}{c}{ Hypothèse Vitesse $-9 \%$} \\
\hline $\begin{array}{c}\text { Distance effectuée } \\
\text { dans Paris (Km) }\end{array}$ & 3 & 5 & 7 & 3 & 5 & 7 \\
\hline $\begin{array}{c}\Delta \text { TEMT } \\
\text { \% TEMT 2000 }\end{array}$ & -42474 & -64603 & -94911 & -38278 & -54896 & -84603 \\
Coût (Mds $€$ ) & $-5,9$ & $-8,2$ & $-11,6$ & $-5,0$ & $-7,0$ & $-10,4$ \\
$\quad$ \% PIB 2004 & $-1,2$ & $-1,6$ & $-2,3$ & $-1,0$ & $-1,4$ & $-2,1$ \\
\hline
\end{tabular}

Source : calculs des auteurs

En retenant le temps de transport domicile travail moyen effectué en voiture (30 minutes) et selon la distance parcourue dans Paris, nous estimons entre 5,5 et 10,9 milliards d'euros la perte annuelle engendrée par l'inflexion de la politique parisienne des transports au niveau de la région Île-de-France (entre 1,2 et 2,3\% du PIB régional).

Un coût de 7,7 milliards (1,6\% du PIB régional) estimé sur la base de la distance effective de cinq kilomètres parcourue dans Paris nous semble la plus proche de la réalité. Cette évaluation s'appuie sur l'hypothèse d'une diminution de $12 \%$ de la vitesse de circulation entre 2000 et 2004. 
L'absence de consensus autour de cette évolution de la vitesse nous incite à la prudence dans l'interprétation de nos résultats. Certains l'estiment en effet surévaluée au regard de la diminution annoncée du trafic automobile. Or, nous savons que les données relatives à la circulation automobile dans Paris ne transcrivent qu'une partie de la réalité qui se limite à celle du "réseau instrumenté ${ }^{19}$. Les statistiques officielles ne font aucunement référence à l'incidence supposée d'un effet de déport de la circulation sur le réseau « secondaire », ni de son impact sur la vitesse moyenne des déplacements.

Une étude de sensibilité nous permet d'évaluer le niveau de confiance à accorder à nos estimations. En nous appuyant sur une hypothèse de réduction de la vitesse moyenne de circulation de $9 \%$ intervenue entre 2000 et 2004, les évolutions observées sont du même ordre de grandeur que précédemment ${ }^{20}$. Selon la distance parcourue dans Paris, la taille effective du marché du travail en 2004 est inférieure de 5 à 10,4\% à ce qu'elle était en 2000. Le coût estimé s'élève entre 4,7 et 9,8 milliards d'euros par an (entre 1 et $2,1 \%$ du PIB régional).

\section{$\mathrm{V}$ - CONCLUSION}

L'objet de cette contribution consiste à évaluer le coût de la politique parisienne des transports introduite en 2001 compte tenu de ses effets sur l'accessibilité de l'emploi régional.

L'impact de la réduction de l'espace viaire alloué à l'automobile sur les conditions d'appariement de la main-d'œuvre a jusqu'alors été totalement occulté. Nos évaluations révèlent pourtant son poids significatif sur l'économie francilienne de l'ordre de $1,5 \%$ du PIB.

La réduction affichée de la circulation ne s'accompagne en aucun cas d'une amélioration de la mobilité et de l'accessibilité des emplois, contrairement aux objectifs affichés. La politique parisienne participe même la dégradation des conditions d'accès à l'emploi à l'échelle régionale.

Les effets de la politique parisienne des transports sont d'autant plus importants que la périurbanisation croissante des emplois et l'atonie des investissements sur le réseau structurant de transports en commun depuis près de vingt ans se traduisent par un changement modal qui se manifeste par un glissement du mode lent vers les modes rapides de transport (automobile, véhicule deux roues motorisé).

Durant la dernière décennie, le ralentissement de l'activité économique en Île-deFrance s'est traduit par une diminution du nombre total de déplacements pendulaires domicile-travail (-67 000), tandis que ceux effectués en voiture augmentaient parallèlement de près de 14000 .

Alors que l'usage de la voiture est de plus en plus contraint, l'évolution de la structure régionale en génère un besoin croissant qui souligne l'insuffisance des infrastructures de transports en commun.

\footnotetext{
${ }^{19}$ Le « réseau instrumenté » ne couvre que 185 kilomètres de voirie en 2001 (190 km en 2004), soit $10,9 \%$ des $1700 \mathrm{Km}$ de voies publiques de circulation.

${ }^{20}$ L'hypothèse d'une réduction de la vitesse moyenne de circulation de $9 \%$ semble corroborée par les dernières données rendues publiques. La municipalité parisienne fait état, pour l'année 2005, d'une vitesse moyenne de $15,9 \mathrm{Km} / \mathrm{h}$ contre $15,8 \mathrm{Km} / \mathrm{h}$ d'après notre estimation.
} 


\section{BIBLIOGRAPHIE}

ACEMOGLU, D. (1996), A microfoundation for social increasing returns in human capital accumulation, Quarterly Journal of Economics, 111, 3, 779-804.

CERVERO, R. (1996), Jobs-housing balance revisited: trends and impacts in the San Francisco Bay Area, Journal of the American Planning Association, 62, 4, 492-511.

CERVERO, R. (2001), Efficient Urbanisation: Economic Performance and the Shape of the Metropolis, Urban Studies, 38, 10, 1651-1671.

Direction Régionale de l'Equipement d'Île-de-France (DREIF), Les déplacements des franciliens en 2001-2002, Enquête Globale de Transport.

INSEE (1999) « Recensement Général de la Population ».

KAIN, J. (1993), The spatial mismatch hypothesis: three decades later, Housing Policy Debate, 3, 371-460.

PRUD'HOMME R., KOPP P. et BOCAREJO, J.P. (2006), Evaluation économique de la politique parisienne des transports, Transports, $\mathrm{n}^{\circ} 434$, pp. 346-359.

PRUD'HOMME, R. (1999), Estimating the Benefits of the Rehabilitation of Sao Paulo downtwon, working paper, Banque Interaméricaine de développement (IADB).

PRUD’HOMME, R, HUNTZINGUER H., KOPP P. (2004), Stronger Municipalities for Stronger Cities in Argentina, working paper, Banque Interaméricaine de développement (IADB).

PRUD'HOMME, R. et LEE C-W (1999), Size, Sprawl, Speed and the Efficiency of Cities, Urban Studies, 36, 11, 1849-1858.

SIMPSON, W. (1992), Urban Structure and the Labour Market: Worker Mobility, Commuting, and Underemployment in Cities. Oxford: Clarendon Press.

ZAHAVI, Y. (1979), The UMOT project, US Department Of Transportation, Washington, DC.

ZAHAVI, Y. et TALVITIE, A. (1980), Regularities in Travel Time and Money Expenditure, Transportation Research Record, 750, 13-19. 